\title{
Different sound decay patterns and energy feedback in coupled volumes
}

\author{
Hongjie $\mathrm{Pu}^{\mathrm{a})}$ and Xiaojun Qiu \\ Key Laboratory of Modern Acoustic, Institute of Acoustics, Nanjing University, Nanjing 210093, China \\ Jiqing Wang \\ Institute of Acoustics, Tongji University, Shanghai 200092, China
}

(Received 16 January 2009; revised 18 January 2011; accepted 19 January 2011)

\begin{abstract}
Different non-exponential decays such as the concave and the convex double sloped decays in the coupled rooms provide distinct sound qualities. These are commonly considered to occur in the less reverberant sub-room and the more reverberant sub-room, respectively. However, numerical simulations and experiments in this paper show that the demarcation line is not located along the physical boundaries (e.g., the partition and the coupling aperture), but in the more reverberant sub-room. The sound field with the concave double sloped decay penetrates into the auxiliary sub-room to an extent which is influenced by the difference between the two natural reverberations of the subrooms. Furthermore the sound energy flows in different regions are investigated, demonstrating how energy feedback leads to the concave double sloped decay.
\end{abstract} (C) 2011 Acoustical Society of America. [DOI: 10.1121/1.3553223]

PACS number(s): 43.55.Br, 43.55.Ka [LMW]

Pages: 1972-1980

\section{INTRODUCTION}

Coupled rooms have been attracting considerable attention in recent decades because the double sloped decay (DSD) in the rooms might provide a compromise between the competing sound qualities of clarity and reverberation. ${ }^{1}$ A DSD has different decay rates in early and late stages. Particularly, the concave DSD has a rapid initial decay rate and a slow one in the tail, which is believed to be able to provide good sound quality. Subjective evaluations show that people can definitely distinguish the DSD from the exponential curve or even DSD curves with different degrees. ${ }^{2-4}$ Ermann conducted subjective testing using exponential and nonexponential decays to determine preference of DSD vs single slope decays, and the results indicated that people can distinguish between DSD and single slope decays but no certain results on preference was obtained. ${ }^{5}$ More recently, Bradley and Wang conducted subjective testing in the coupled rooms with different architectural parameters. By using multidimensional scaling analysis, they found that people seem to prefer low and middle levels of double sloped effect. ${ }^{6}$

According to prior work, ${ }^{7-11}$ two types of non-exponential decay curves can exist in the coupled rooms, the concave DSD and the convex DSD. The concave DSD has a fast early decay rate and a slow late decay rate, while the convex DSD is the opposite, decaying slowly at first then fast in the tail. The concave DSD always appears in the less reverberant sub-room which has also the source in it, and the convex DSD can be found in the other sub-room. In the classical statistical acoustic theory, each of the two different shapes of decay curves only exists in its own room, so the demarcation line is at the coupling aperture. ${ }^{7}$ However, as the require-

\footnotetext{
a) Author to whom correspondence should be addressed: Electronic mail: puhongjie@nju.edu.cn
}

ments for the classical statistical acoustic theory might not be met in practice, it would be of interest to know where the true demarcation line is located.

On the demarcation line of sound fields in the coupled rooms, some prior research can be traced. In 1925, Davis ${ }^{7}$ proposed statistical acoustics (SA) theory, in which ordinary differential equations were solved to describe the sound energy density's decay in the coupled rooms under the ideal conditions of uniform diffusion in each sub-room and an abrupt transition at the coupling aperture. In 1931, Eyring ${ }^{8}$ improved Davis' original SA model with the Eyring's absorption coefficient and a proper correction for frequencyweighted absorption on the coupling aperture. In both models, the ideal condition of the abrupt transition at the coupling aperture was assumed, and it was also mentioned that the abrupt transition assumption breaks down in practical instances. They suggested that the shapes of decay curves near the coupling aperture would change and have a smooth transition through it. But as both SA models are lumped parameter systems, no more details about the spatial variation have been given.

In a recent paper, Summers et al. ${ }^{12}$ proposed a modified SA model which can partially represent the spatial variation. By generating "secondary sources" on the coupling aperture and making the sources radiate in the Lambertian style, the spatial variation of steady sound energy density was obtained. But the ordinary differential equations' eigenvalues make the decay constants the same all over each sub-room, so the transition of decay curves' shapes can hardly be observed with this method. Four years later, by simulating the energy flows in several points in the coupled rooms with the diffusion model, Jing and Xiang discussed the phenomenon of energy feedback ${ }^{13}$ and revealed a "reversal" characteristic of energy flow directions and its dependency on the size and location of the aperture. 
Nowadays, many numerical methods can be used to estimate the sound field in the coupled rooms, like SA, ${ }^{7,12,14}$ the classical wave method, ${ }^{15,16}$ geometrical acoustics (GA), ${ }^{17-20}$ and the diffusion equation model (DEM). ${ }^{21-23}$ To obtain the proper information of spatial variation for both steady state values and decay constants, only the ray-tracing and DEM methods (essentially an extension of SA) are employed in this paper. For analyzing decay curves, the DSD model and Bayesian parameter estimation are used to quantify the parameters $^{24}$ because it is more reliable than fitting decay curves with two straight lines. ${ }^{25}$

In the research, a simple coupled system with only two rectangular sub-rooms connected with a square aperture was used. The decay curves over the coupled rooms were estimated by the ray-tracing method, the diffusion model method, and experiments. By quantifying the curves' curvatures, a demarcation line of different decay patterns was found. The relationship between its position and the absorption was also investigated. Finally, the energy fluxes in the coupled rooms were examined to explain the mechanism of DSD's occurrence.

\section{MODEL AND METHODS}

\section{A. Geometry of the coupled rooms}

The coupled rooms used in this paper have dimensions of $5 \times 8.25 \times 4.75 \mathrm{~m}^{3}$ as illustrated in Fig. 1. An aperture was opened between the main volume (sub-volume I) and the auxiliary volume (sub-volume II). The aperture has a size of $1.25 \times 1.25 \mathrm{~m}^{2}$. The origin of the coordinate system was at the left upper bottom corner in the main sub-volume as shown in Fig. 1 and a point sound source was located at $4.0,1.0,1.0 \mathrm{~m}$. The main room was $50 \%$ smaller than the auxiliary room, which insured that the natural reverberation time estimated by the Eyring equation could be sufficiently larger than that in the main room, so that the effects of the DSD and the energy feedback could be observed clearly.

The acoustic fields in both the main and the adjunctive sub-volumes were calculated. By choosing 77 points around

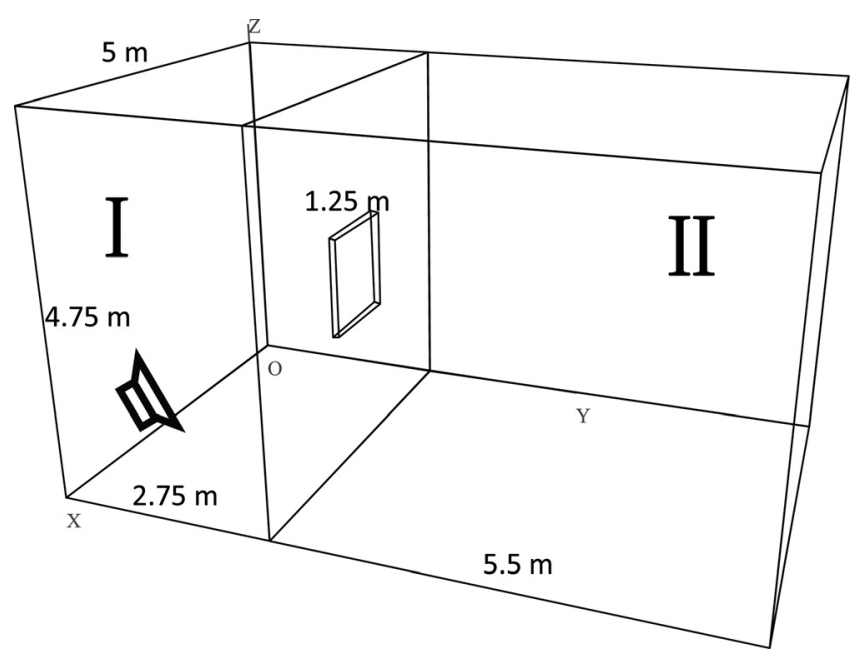

FIG. 1. (Color online) Geometrical configuration of the coupled volumes. Sub-volume I $\left(5 \times 4.75 \times 2.75 \mathrm{~m}^{3}\right)$ in the left, and sub-volume II $\left(5 \times 4.75 \times 5.5 \mathrm{~m}^{3}\right)$ in the right, with a coupling aperture of $1.25 \times 1.25$. the coupling aperture in the plane of half height and analyzing the impulse responses at these points, a further investigation to the mechanism of acoustical coupling at these points around the coupling aperture was carried out.

Figure 2 shows the arrangement of the receiving points. Thirty-six receiving points were located in the main sub-volume, which were arranged in four lines. The distance between each line was $0.25 \mathrm{~m}$ and the distance between points along the same line was $0.5 \mathrm{~m}$. Another four lines of receiving points were located in the adjunctive sub-volume. The arrangement was the same as that in the main subvolume except that the distance between the last three lines was changed to $0.5 \mathrm{~m}$. Five additional points were located at the coupling aperture with a spacing of $0.25 \mathrm{~m}$.

To obtain different coupling configurations of natural reverberation between the sub-volumes, 11 different sets of absorption coefficients from 0.05 to 0.95 were assigned to the main sub-volume while keeping the assignment in the auxiliary sub-volume unchanged at 0.05 . In this way, the relationship between the DSD and the absorption coefficient was investigated.

\section{B. Simulation methods}

The ray-tracing method and the diffusion model method were used for the numerical simulations. A commercial software ODEON has been applied to do the ray-tracing simulations, which is a combined model of the image source method and the ray-tracing method. To ensure the accuracy of simulations in the couple rooms, $10^{6}$ rays were used in ODEON and the upper limit of reflection order was set to 2000 . The melamine faced synthetic wooden board was set with a scattering value of 0.1 and other rough absorption materials were set with a value of 0.5 . The scattering was ruled by the Lambert's scattering as the controlling function. The recording files of the ray tracing were extracted from ODEON, which can provide the information of rays' intensities and directions at the observation point every time. The ray denotes the energy flux and it is equivalent to the mean active sound

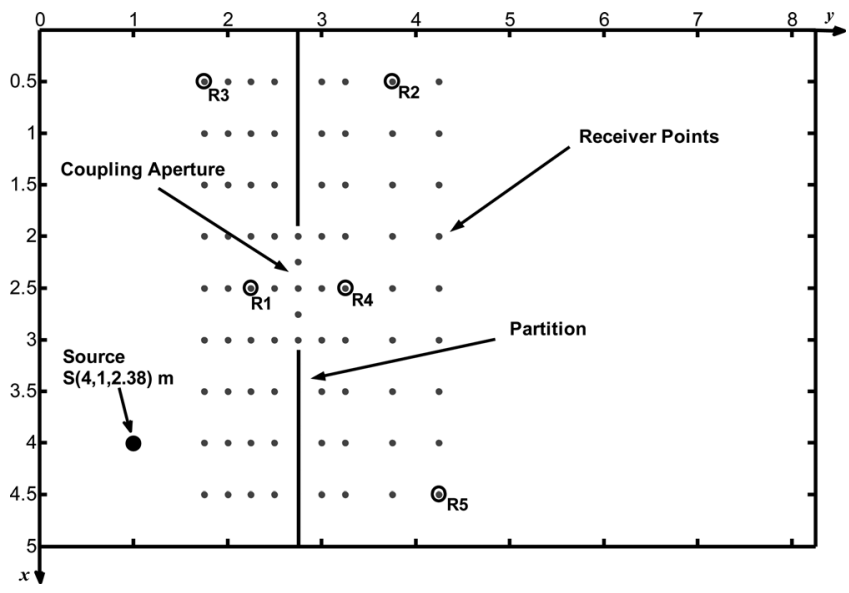

FIG. 2. The arrangement of the receiving points in the coupled volumes at the plane of half height, with 36 receiving points in sub-volume I, 36 receiving points in sub-volume II, and 5 receiving points on coupling aperture. Among them, the decay curves and sound flows at five typical points (R1, R2, R3, R4, and R5) are discussed in details. 
intensity in middle and high frequencies. The component of the sound energy flux in the $j$-axis direction of Fig. 1 was calculated particularly. The steady state and decay of energy flux were calculated by using backward integration method for sound intensity.

The diffusion function used in the DEM is expressed as, ${ }^{21}$

$$
\frac{\partial w(\vec{r}, t)}{\partial t}-\frac{\lambda c}{3} \nabla^{2} w(\vec{r}, t)+m w(\vec{r}, t)=q(\vec{r}, t),
$$

where $w(\vec{r}, t)$ is the density of sound energy, $q(\vec{r}, t)$ is the power of sound sources, $m$ is the coefficient of air dissipation, and $\lambda$ is the mean-free path. By calculating the gradient of $w(\vec{r}, t)$, the energy flow (or the real part of the complex sound intensity) can be obtained as,

$$
I=-\frac{\lambda c}{2} \operatorname{grad} w(\vec{r}, t)
$$

The diffusion equation was numerically solved. For convenience, the entirety of the coupled volumes was uniformly meshed in cubic form for a total of 25600 units, and Eq. (1) was chosen as the control equation in the solving process. By applying Eq. (2) to the solved results and extracting the component in the $j$-axis, the distribution of sound intensity in the coupled rooms was obtained.

When the solving procedures were applied, two steps were arranged to obtain the decay process. The first step was to solve the diffusion equation's partial differential equations with a steady exciter and zero initial values for all subdomains. Then the steady state of the sound field was obtained, which was used in the second step. In the second step, a zero-exciter response solution was calculated with the initial values assignment of the steady sound energy density distribution solved in the first step. This solution can be considered as a strict solution for the decay process and has no "mixing process" at the beginning which has been mentioned by Valeau. ${ }^{23}$

\section{The experimental method}

In the experiments, a 1:5 scaled model was used as shown in Fig. 3. As with the numerical simulations, the source was located at the corner of the main volume. A single loudspeaker box with a dimension of about $15 \mathrm{~cm}$ was employed as the source in the experiments. Although it was somewhat directional at about $5 \mathrm{kHz}$, it was placed facing the corner to make the radiation sufficiently uniform after the first reflection. Seventy seven points around the coupling aperture were selected, which were arranged in nine rows including the aperture itself.

The maximum length sequence and fast Hadamard transform were applied to obtain the impulse responses at these 77 points, which were the acoustical transfer functions from the source point to these points. The built-in maximum length sequence module of Nor-sonic 840 was used to achieve the measurements. The impulse responses were exported and filtered with an octave band-pass filter at the center frequency of $5 \mathrm{kHz}$. After applying the Schroeder backward integration technique to these octave band-pass

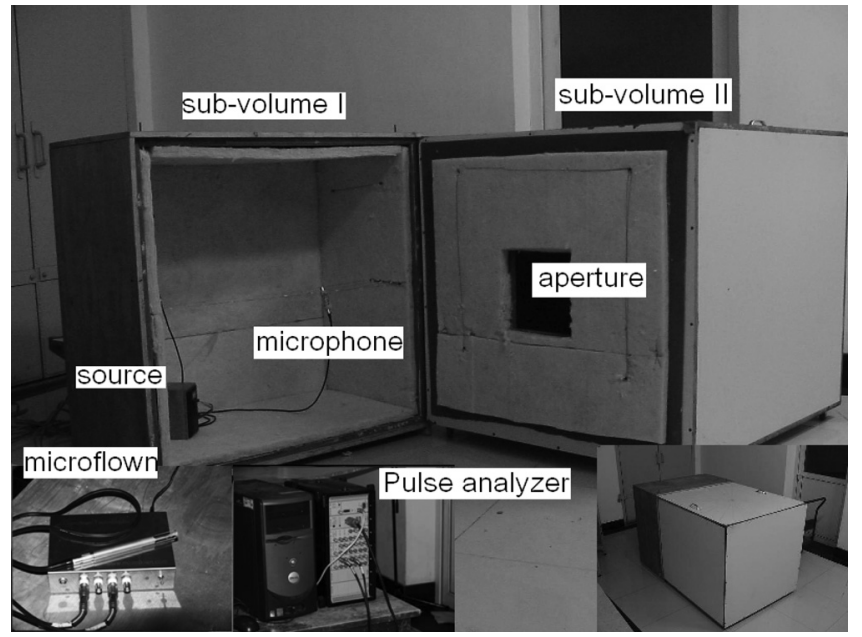

FIG. 3. The 1:5 scaled model of the coupled rooms, with a Microflown Universal Kit and a 16-channel B\&K ulse analysis system.

filtered impulse responses, ${ }^{26}$ the decay curves at the 77 points were obtained. The recorded decay curves were then analyzed using the decay model and the Bayesian parameter analysis which will be depicted in Sec. II D.

For sound energy flow measurements, a Microflown Universal Kit was used as shown in the bottom left of Fig. 3. ${ }^{27}$ The Microflown probe can provide signals for both sound pressure and particle velocity. After pre-amplification and phase correction, the signals were collected by a 16-channel B\&K Pulse system which was shown in the bottom of Fig. 3 . For the measurements, a white noise signal was generated by the Pulse system to establish the acoustical field in the model. After the field became stable, the generator was turned off and the whole decay duration was recorded.

The constant percentage bandwidth analysis was applied to both sound pressure and particle velocity signals. To obtain the instantaneous sound intensity, real-time cross-correlation was processed on the spectrum of these two signals, from which the real part was the sound intensity needed. ${ }^{28}$ Because of the random nature of white noise in time, an averaging time of $1 / 64 \mathrm{~s}$ was chosen in the constant percentage bandwidth analysis, which helped to obtain the smooth SI curves. Otherwise, more than 50 averages should be required to obtain a comparable result.

Two different materials were used in sub-room I; one was a sponge layer with $5 \mathrm{~mm}$ thickness and the other was a polymer foam layer with $30 \mathrm{~mm}$ thickness. The sponge has an absorption coefficient of about 0.60 in the $5 \mathrm{kHz}$ octave band and the foam's is about 0.88 . The results of these two materials were used to support the conclusions drawn through the numerical results. In describing the energy exchange between the coupled rooms, only the $5 \mathrm{~mm}$ sponge was used to illustrate where and how sound energy feedback occurred.

\section{Analysis and quantification}

To analyze the decay curves obtained in the simulations and experiments, the decay model proposed by Xiang et $a l .{ }^{24}$ was used. The energy decay function of a selected point can be expressed as 


$$
E\left(A, \delta, t_{k}\right)=\sum_{j=1}^{m} A_{j} G_{j}\left(\delta_{j}, t_{k}\right)
$$

where $G_{j}\left(\delta_{j}, t_{k}\right)=\left\{\begin{array}{ll}e^{-\delta_{j} t_{k}} & j \neq m \\ L-t_{k} & j=m\end{array}\right.$. It contains several components of exponential decays and a residual linear decay of additive background noise. $A_{j}$ is the initial value of each decay mode; $\delta_{j}$ is the decay constant of each exponential decay mode; $L$ is the steady state noise level. It is a general expression at any position of an inner space with background noise, no matter whether diffused or not throughout the coupled spaces. In a weakly coupled system, such as when the coupling aperture is much smaller than the partition, $\delta_{j}$ keeps the natural damping property of each sub-volume. Although a decay function for a multi-coupled system can be drawn theoretically, it is hard to find more than two damping constants $(m>3)$ in real-measured decay curves. ${ }^{29}$

As presented above in the decay model, the curvature can be depicted with "decay ratio" $D R=\delta_{1} / \delta_{2}$, defined as the ratio of non-linear parameters, which are the damping constants of the first and second decay mode in the decay model. The value of DR describes the apparent curvature of the decay curves. For values of $D R>1$, an obvious concave DSD can be observed, while the curves with $D R<1$ can be called convex DSD. Consequently $D R=1$ is the demarcation line between sound fields with these two different decay patterns. For acousticians, it might be useful to demarcate the sound field in the coupled rooms by different decay patterns other than according to the geometrical configurations. So the sound field was quantified with the parameter $D R$ in this paper to identify whether a concave DSD exists or not and where it exists.

The linear parameters in the decay model of Eq. (3) suggest an energy exchange. By fixing the value of $A_{1}$ positive, the sign of $A_{2}$ denotes the direction of energy flow at the position where this curve is obtained. If is positive, it means that there is an energy feedback from somewhere else to slow down the damping speed of energy density in the local area; and if $A_{2}$ is negative, it is the opposite and an extra energy that flows out to other areas beside damping itself. In practical cases, energy feedback usually occurs with the concave DSD of value $D R>1$.

Fitting the decay curve with the double decay model is one type of non-linear fitting method, where the indicator "decay ratio" can be calculated to quantify the curvature of non-exponential decay. Some other indicators from linear fitting such as EDT/LDT (LDT means late decay time), $\mathrm{T}_{10} / \mathrm{LDT}$, and $\mathrm{T}_{15} / \mathrm{T}_{30}$ are often used in quantifying curvature as in Bradley and Wang's investigation. ${ }^{6}$ If a truly diffuse sound field can be realized in the coupled rooms, like the diffusion model in simulations, then the model is fit well and can reveal the coupling mechanism of energy exchange. However, when applying curve fitting, one should be cautious about the validity of the DSD model as well as other linear fitting methods.

\section{RESULTS}

\section{A. Different decay patterns}

Two distinct types of decay curves exist in the coupled rooms. The concave DSD curves can usually be observed in the sub-room with a source in it, while the convex one can be found in sub-rooms without a source in it. Generally speaking, the concave decay has a visible shift in curvature, so it can be distinguished from the exponential decay curves with naked eyes. The convex DSD curve is harder to distinguish except for a zero initial slope. A correction for the delay between the source and the receiver was made in the simulations and experiments to remove its influence on the early part of decay curves. The initial portion of the obtained room impulse response (RIR) was removed up to a length of time $\Delta t=\frac{d}{c}$, where $d$ is the distance between the source and receiver and $c$ is the sound speed in air. If this delay is kept in the RIR, it makes the initial part of a decay curve flat when the RIR is backward integrated, which can lead to a mistake in distinguishing whether it is a convex DSD or just an exponential decay with an additive steady beginning.

All results discussed below are for the octave band with a center frequency of $1 \mathrm{kHz}$ ( $5 \mathrm{kHz}$ in the scale models). Figure 4 shows the two different types of non-exponential decay curves, where Fig. 4(a) is the decay curves obtained by three different approaches in the main room at the position R1 (2.50, 2.25, $2.38 \mathrm{~m})$ and Fig. 4(b) gives the convex decay curves in the adjunctive sub-room without a source in it at the position $\mathrm{R} 2(0.50,3.75,2.38 \mathrm{~m})$. The solid line is the result of the diffusion model, the dashed line is the result from ODEON (ray-tracing method), and the dot line is the experimental result.

The sound energy in Fig. 4(a) damps rapidly at the beginning and slows down in the later part. The early part provides a short early decay time (EDT) to the audience, while the later part provides a relatively long reverberation. In Fig. 4(b), all the curves start with an initial slope of zero. In most instances, the convex DSD curves are not as remarkable as the concave ones.

The dash-dotted lines in Fig. 4 are curves generated with Bayesian parameters, which were obtained by estimating the decay curves calculated with the diffusion model. The parameters of these two curves are illustrated in Table I. It is presented here only for the purpose of giving an example of concave and convex DSDs, so only some estimated parameters are listed in Table I. However, all the decay curves are analyzed in the simulations and experiments with the decay model in Eq. (3), and the estimated parameters are presented in the form of "decay ratio" to quantify and describe the difference of decay shapes in different regions of the coupled rooms.

\section{B. Demarcation line}

As discussed above, two different patterns of decay can be observed in the coupled rooms. By quantifying the decay curves with the decay model and calculating the decay ratios of these curves, a series of contour lines were plotted in Fig. 5. In this case, the sub-room I was covered by the material with an absorption coefficient of 0.6. 
(a)

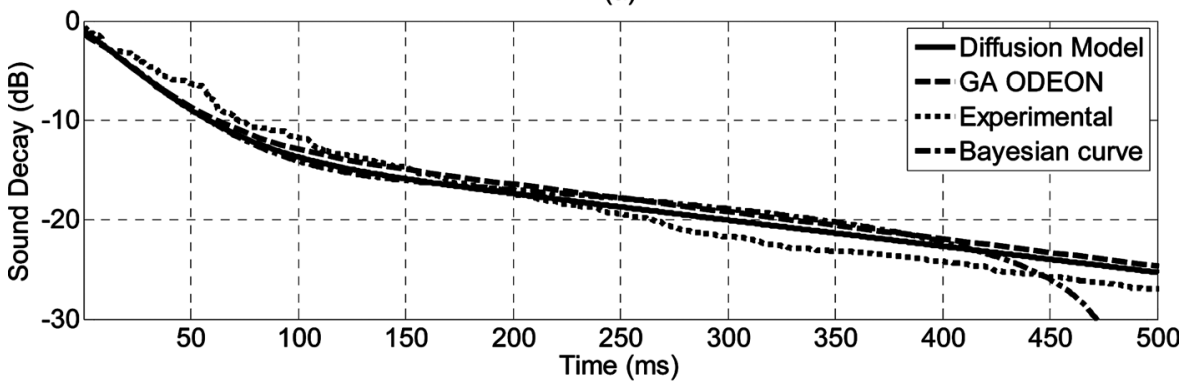

(b)

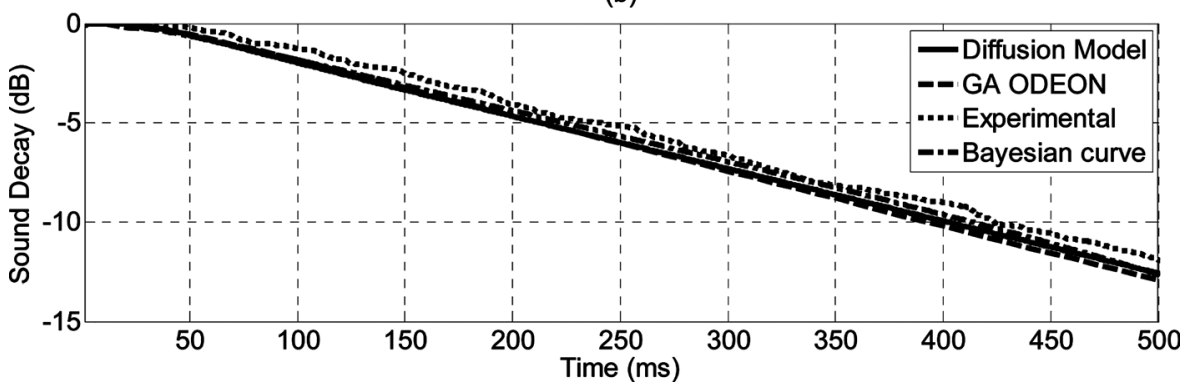

FIG. 4. Two types of sound decay curves obtained with the diffusion model, the raytracing method and experiments: (a) concave DSD curves at R1 $(2.50,2.25,2.38 \mathrm{~m})$ and (b) convex DSD curves at R2 (0.50, $3.75,2.38 \mathrm{~m})$. The absorption coefficient in sub-volume I equals 0.6. Solid line is for the diffusion model method, dashed line for the ray-tracing method, dotted line for experiments, and dash-dot line for curves generated by the Bayesian parameters.
Figure 5(a) is the contour map of the decay ratio calculated with the ray-tracing method, where the contour lines with values of 3,2 , and 1 are plotted. As expected, the decay ratio of decay modes in sub-volume $\mathrm{I}$ is high value, but the decay ratio at some points in sub-volume II is also larger than 1. This has not been well explained by the classical SA theory. It is clear in the figure that the sound field with the decay ratio value over 1 permeates from sub-volume I into sub-volume II. The line of $D R=1$ separates the sound field with different decay patterns, and the line might not be along the physical partition or the coupling aperture.

Figure 5(b) shows eight decay curves at eight points from coordinate $2.5,1.75,2.38 \mathrm{~m}$ to $2.5,4.25,2.38 \mathrm{~m}$ with a spacing of $0.36 \mathrm{~m}$ along $y$-direction at the center of the coupling aperture, and the gradual change of these curves indicates the sound field permeation phenomenon. Figures 5(c) and 5(d) are the results calculated with the diffusion model. The same permeation can be observed; however, it seems that the diffusion model is less sensitive than the ray-tracing method to the effects of the barriers (it is the partition here) between the source and receivers. ${ }^{21}$

Figure 6 compares the results obtained with different methods when the sub-volume I has an absorption coefficient of 0.6. From the demarcation line of $D R=1$, it is clear that the sound field with the concave decay property goes from sub-volume I to II through the coupling aperture. According to the experimental results, the concave DSD can barely be found in the space just behind the partition in sub-volume II, and it is still dominated by the exponential or convex decay.

TABLE I. Bayesian parameters of solid decay curves (estimated with the diffusion model) in Fig. 4.

\begin{tabular}{lrrrrrr}
\hline \hline & \multicolumn{1}{c}{$\delta_{1}$} & \multicolumn{1}{c}{$\delta_{2}$} & $A_{1}$ & \multicolumn{1}{c}{$A_{2}$} & $A_{3}$ & \multicolumn{1}{c}{$D R$} \\
\hline Fig. 4(a) & 42.27 & 6.22 & 1.36 & 0.05 & 0.10 & 6.80 \\
Fig. 4(b) & 6.08 & 48.68 & 1.25 & -0.19 & 0.10 & 0.13 \\
\hline \hline
\end{tabular}

The discrepancy between the ray-tracing and the experimental results might be caused by the diffraction in the experiments. In the scale model, the coupled aperture has a size of $0.25 \times 0.25 \mathrm{~m}$, the considered frequency band ascends from 1 to $5 \mathrm{kHz}$, and the wavelength is now about $0.05 \mathrm{~m}$. Under this condition, the diffraction of the edge cannot be neglected in the shadow zone. However, because the direct sound can reach other areas, the reverberant sound density is lower there than that in the shadow zone. This is equivalent to the shadow zone having relatively high reverberation, so the convex DSD appears more preponderant in this area. In the ray-tracing and diffusion models, though, no diffraction is considered. This might be the reason that the discrepancy around the shadow zone occurs.

\section{Effects of absorption}

As presented in Sec. III B, the concave DSD in subvolume I permeates into the sub-volume II. The degree of permeation can be quantified with the percentage of the concave DSD volume to that of the whole sub-volume II. As a rigorous volume calculation of the permeation cannot be obtained easily, only the area of inleakage on the half height plane was investigated. Figure 7 shows the percentage of the concave DSD area in sub-volume II as a function of absorption coefficient in sub-volume I. The solid line is the result calculated with the diffusion model method and the dashed line is that with the ray-tracing method. It can be observed from the figure that these two methods show the same trend: The percentage of inleakage increases with the increase of absorption in the sub-volume I. The experimental results are closer to that of the ray-tracing method; however with slightly larger values. For the absorption coefficient of 0.6 , about $5 \%$ error exists between the ray-tracing method and the experiments, and this error increases to about $25 \%$ for the absorption coefficient of 0.88 . 
(a)

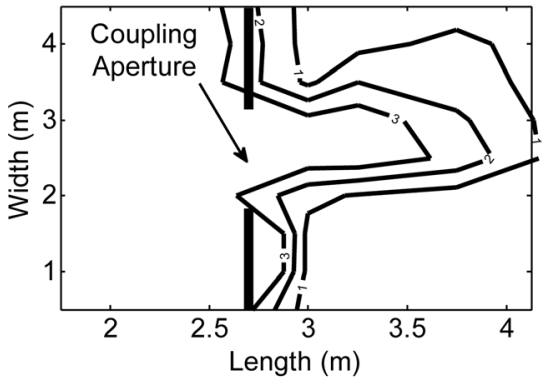

(c)

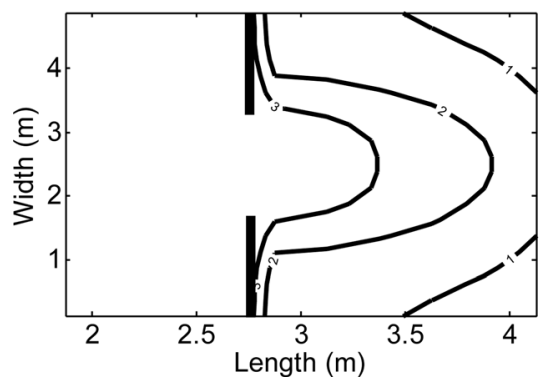

(b)

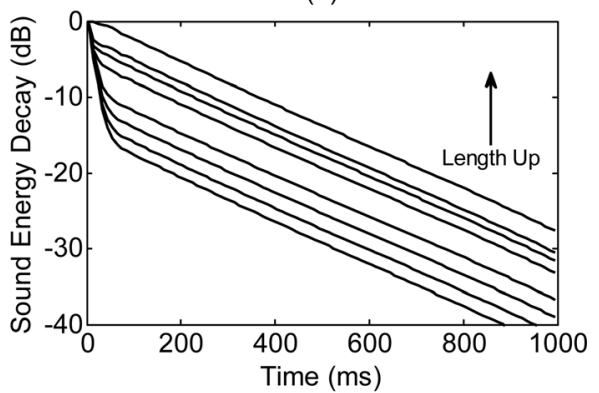

(d)

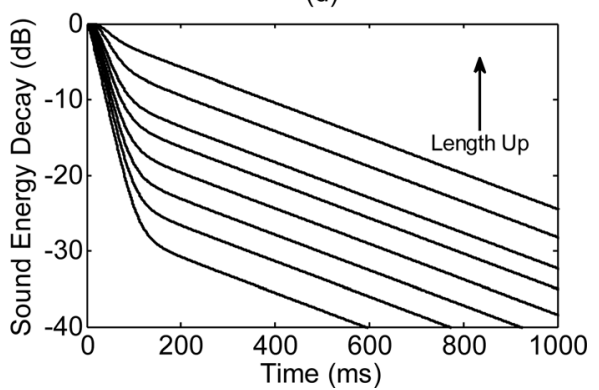

FIG. 5. Contour maps for the decay ratio and decay curves along a line through coupling aperture from coordinate 2.5, 1.75, $2.38 \mathrm{~m}$ to $2.5,4.25,2.38 \mathrm{~m}$, (a-b) for the ray-tracing method and (c-d) the diffusion model method. The direction of arrow "length up" indicates the direction from sub-volume I to sub-volume II.
The percentage of inleakage increases with the increase of absorption coefficient from 0.05 to 0.60 , and after that, no significant increase can be observed. An upper limit point occurs when the absorption coefficient is larger than 0.60. This limit corresponds to about $20 \%$ permeation according to the diffusion model method and $10 \%$ permeation according to the ray-tracing method. The difference of the limits between the two methods is due to the estimating error of the field just behind the partition. The diffusion model always overestimates the sound field just behind the partition, no matter about the steady sound pressure (see Fig. 8 in Ref. 21) or the decay ratio (see Fig. 6).

When the absorption coefficient equals 1 in sub-volume I, the coupled rooms degenerate to the case of an enclosure with an open window to the free space, in which a concave DSD can still be observed.

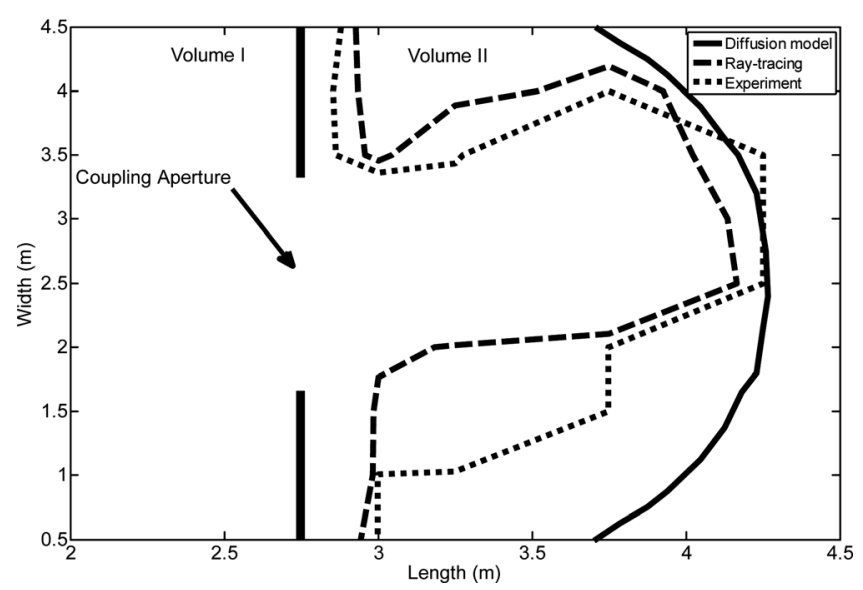

FIG. 6. Comparison of the demarcation line of the sound field in the coupled rooms, where $D R=1$ between results from the DEM method (solid line), ray-tracing method (dashed line), and the experiments (dotted line).

\section{ENERGY FLOWS}

Figure 8(a) shows the time recording of the sound intensity measured at the receiving point $\mathrm{R} 1$ in Fig. 2. The absorption coefficient is 0.6 in the main room and the whole measurement lasted for $15 \mathrm{~s}$. The generator was turned on at about the $1 \mathrm{st} \mathrm{s}$ and interrupted at the 11th $\mathrm{s}$, after which was the decay duration. The sound intensity obtained fluctuated when the generator was just on, and this is because only an average time of $1 / 64 \mathrm{~s}$ is applied in the constant percentage bandwidth analysis. If a longer averaging time was used, the abrupt descending edge was contaminated and hard to be observed, then smoothing and detailing need to be compromised under the current measurement technique because of the random nature of white noise.

Figure 8(b) shows the zoomed decay stage, where the feedback occurs clearly at $11 \mathrm{~s}$ when the sound source was

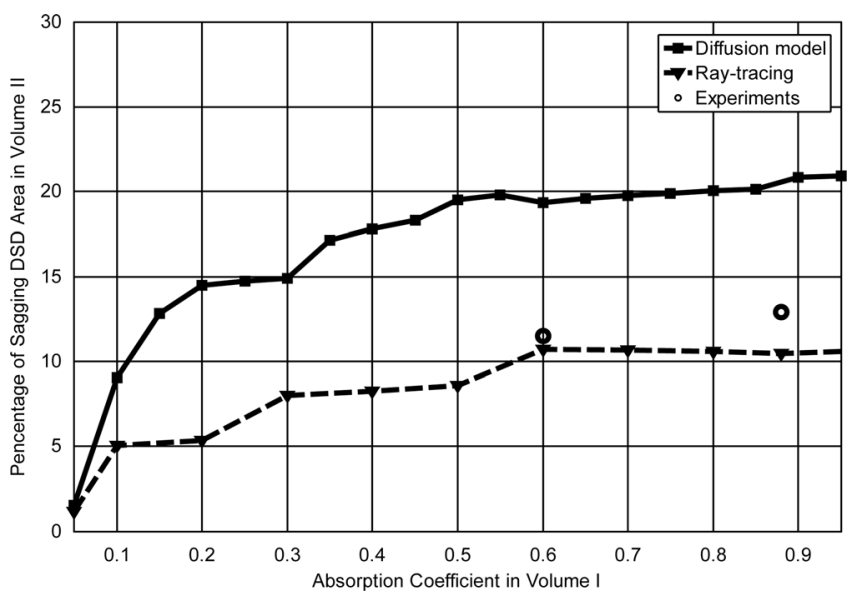

FIG. 7. The degree of permeation of concave DSD in sub-volume II along a horizontal plane as a percentage of area in sub-volume II at two different absorption coefficients $(\alpha=0.60$ and $\alpha=0.88)$ by the DEM method (solid line), ray-tracing method (dashed line), and the experiments (hollow circle). 

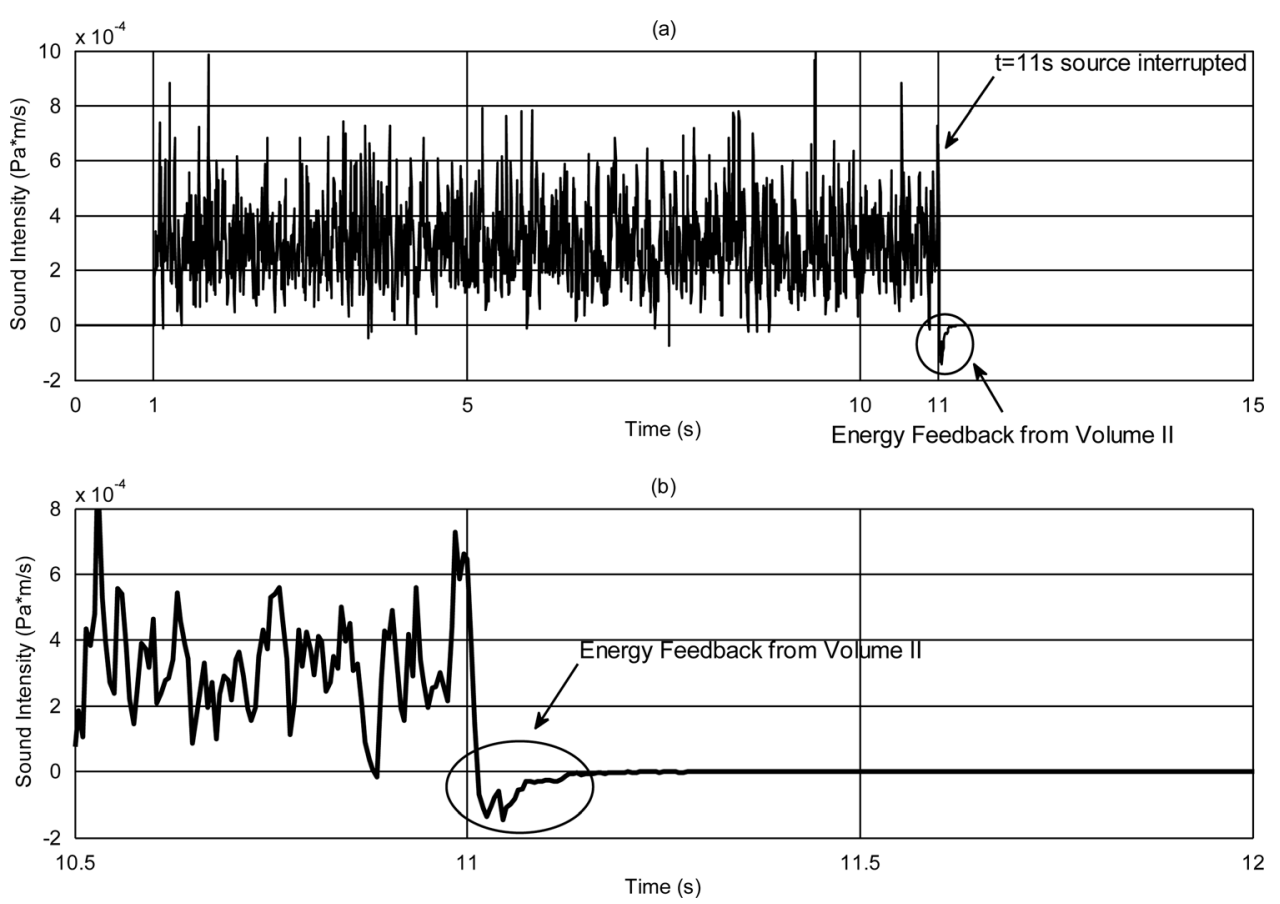

FIG. 8. A typical sound intensity curve as a function of time in the experiment. (a) Plotted across the whole measurement duration and (b) zoomed in around the interrupted point. The absorption coefficient in sub-volume $\mathrm{I}$ is 0.60 and the measurement position is $\mathrm{R} 1$. interrupted. That does not mean feedback exists only after the generator is turned off. In fact, it exists all the time after the generator was turned on; however, it is masked by the intensive energy flow from the source when the source is turned on.

Different types of energy exchange in different areas of the coupled rooms are the mechanisms of different decay patterns. Let $m$ be 3 in Eq. (3) for the general solution of sound energy density in the coupled rooms. The first component $A_{1} e^{-\delta_{1} t}$ denotes the damping of the sub-room itself, the second component $A_{2} e^{-\delta_{2} t}$ denotes the energy exchange introduced by coupling, and the third component $A_{3}(L-t)$ denotes the residual background noise. The part introduced by coupling can be positive or negative according to the classical SA method. A positive value suggests that sound energy feeds back from the other sub-room during the decay process and a negative value indicates that this sub-room or local zone needs to provide extra energy for the other subroom or somewhere else to slow down the damping speed in it. By analyzing the decay curves with different $A_{2}$ values, it can be found that only when $A_{2}$ is positive the concave DSD can be observed. It should be easier to find the phenomenon of energy feedback where both $A_{1}$ and $A_{2}$ are positive in area with $D R>1$, especially near the coupling aperture.

Figure 9 shows three normalized sound intensity curves at the positions of $\mathrm{R} 3(0.5,1.75,2.38 \mathrm{~m}), \mathrm{R} 4(2.5,3.25,2.38 \mathrm{~m})$, (a)

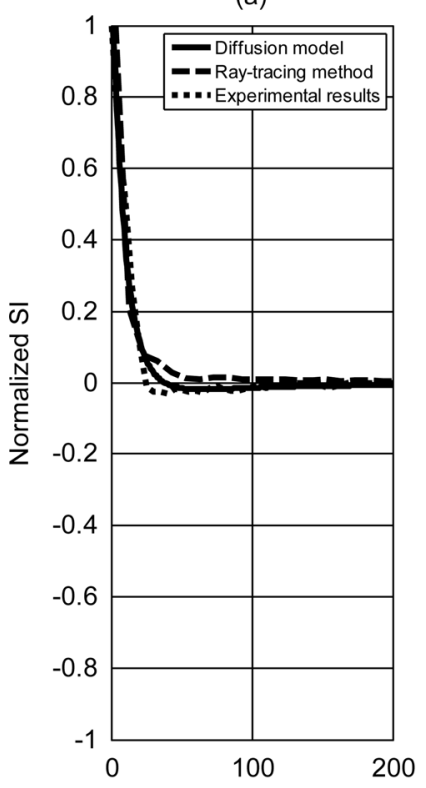

(b)

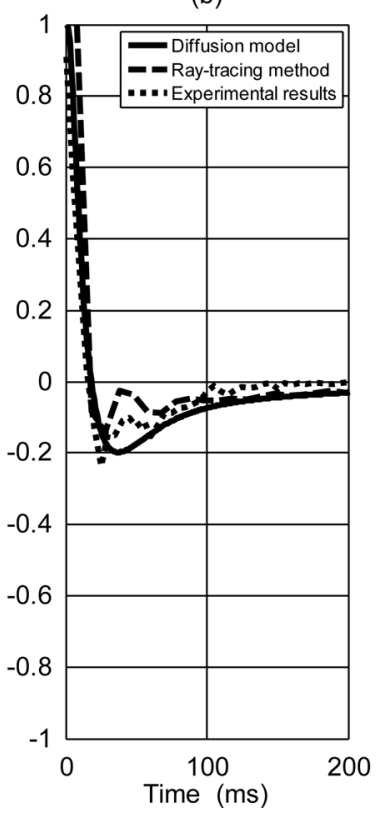

(c)

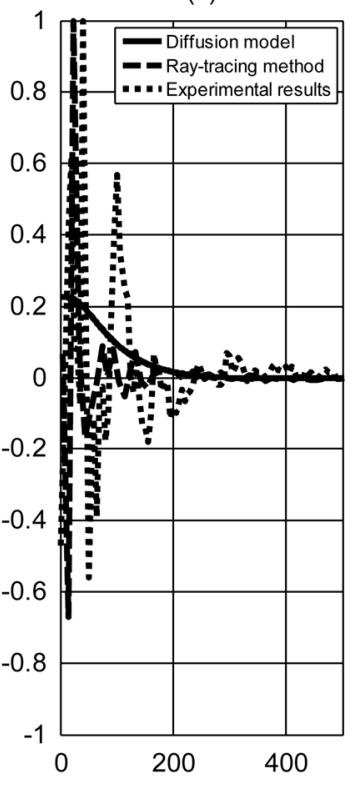

FIG. 9. Normalized sound intensity curves at different position in the coupled rooms (a) R3, (b) R4, and (c) R5, as calculated with the DEM method (solid line), ray-tracing method (dashed line), and the experiment (dotted line). 
and R5 (4.5, 4.25, $2.38 \mathrm{~m})$, respectively. The curves in each figure were normalized by the maximum absolute value during the decay process. The three plots denote the relative amplitudes and directions of energy flows at three different locations in the coupled rooms. The absolute value of the curves denotes the intensity of energy flow and the sign denotes the direction. Positive value means from sub-volume I to II along the $y$-axis as in Fig. 1, and vice versa. The energy exchange is a dynamic process, during which there is energy flow from sub-volume I to II each time as well as in the opposite direction, and the difference between these two parts is the mean active sound intensity that can be observed in the simulation and experiments. The solid lines are the results obtained with the diffusion model method, and the dashed lines are that with the ray-tracing method. The dotted lines are experimental results.

In the area near the inner surface but far away from the coupling aperture in sub-volume I, the direction of energy flow is dominated by the absorption of surfaces. At this place, the observable feedback is extremely small, which is merely $2 \%$ of the steady intensity, as shown in Fig. 9(a). In the area near the coupling aperture, a strong energy feedback occurs, not long after the decay begins, and its intensity reaches about $20 \%$ of the steady energy flow as shown in Fig. 9(b). When the energy flow changes its direction and the absolute value of sound intensity approaches zero, it causes a "pit" in the energy curve when representing the curve on a decibel scale which has even been described by Jing and Xiang. ${ }^{13}$ In the area far away from the coupling aperture in sub-volume II; although the decay curves can be analyzed to be the convex type of DSD, it is difficult to distinguish the decay type from the exponential decay, unless the difference between the absorption coefficients in the two sub-volumes is very large. Energy curves in these areas usually have properties of that in the diffused sound field, in which the direction changes all the time and the strength dampens along with time. In Fig. 9(c), the sound intensity curve calculated with the diffusion model only gives the macro-trend of the energy flow but not the shifting direction, because it is essentially an extension of the SA model and cannot give the detailed movement of each sound particle as the ray-tracing method does. For example, the particle property in the ray-tracing method means that the sound energy transmits discretely along the ray according to the recording interval. At any position in the coupled rooms, sound energy may flow in one direction at this moment, but in another direction at the following moment. So the sound intensity curves estimated with the ray-tracing method seem to be fluctuating.

\section{SUMMARY AND CONCLUSION}

In this paper, the shape of decay curves with time in the coupled rooms was quantified with the parameter "decay ratio." By analyzing the distribution of this parameter in space, a demarcation line was found in the coupled rooms, which divided the whole space into two parts. In each part, a different pattern of DSD could be found. Simulations based on a GA method and the diffusion model method, and scale-model experiments showed that the sound field with concave DSD extended through the coupling aperture to the more reverberant chamber, and the degree of this inleakage depended on the amount of absorption in the primary room. Results showed that the more absorptive it was, the greater was the amount of inleakage. An occupation of about $10 \%$ of the total area in the less reverberant chamber seemed to be the upper limit of the inleakage. Energy flows in areas with different types of decay curves were also investigated. Energy feedback existed during the whole decay process, but it could be observed in the energy curve only when the energy density in the primary room was reduced to a comparative low level. It was the observable feedback energy that made the decay curves non-exponential or even appeared concave. It is recommended that the quantitative relationship of the demarcation line with the aperture size and shape and room absorption coefficients, as well as the relationship between the energy feedback and the change of decay rate might be investigated in future work.

\section{ACKNOWLEDGMENTS}

The authors are grateful to Prof. Lily Wang and the anonymous reviewers for their constructive comments on improving the original manuscript. This work was supported under Projects 11004100 and 11004101 by National Nature Science Foundation of China.

${ }^{1}$ J. Wang, "Diffusion and auditorium acoustics," Build. Acoust. 10, 211219 (2002).

${ }^{2}$ K. S. Sum and J. Pan, "Subjective evaluation of reverberation times of sound fields with non-exponential decays," Acta Acust. Acust. 92, 583592 (2006).

${ }^{3}$ D. T. Bradley and L. M Wang, "Absorption and aperture size effects on subjective response to complex computer modeled coupled volume systems," in Forth Joint Meeting: ASA and ASJ, Honolulu, Hawaii (2006), Paper No. 1pAA12, p. 3011.

${ }^{4}$ D. T. Bradley and L. M. Wang, "The effects of simple coupled volume geometry on the objective and subjective results from nonexponential decay," J. Acoust. Soc. Am. 118, 1480-1490 (2005).

${ }^{5}$ M. Ermann, "Double sloped decay: Subjective listening test to determine perceptibility and preference," Build. Acoust. 14, 91-108 (2007).

${ }^{6}$ D. T. Bradley and L. M. Wang, "Optimum absorption and aperture parameters for realistic coupled volume spaces determined from computational analysis and subjective testing results," J. Acoust. Soc. Am. 127, 223-232 (2010).

${ }^{7}$ A. H. Davis, "Reverberation equations for two adjacent rooms connected by an incompletely soundproof partition," Philos. Mag. 50, 75-80 (1925).

${ }^{8}$ C. F. Eyring, "Reverberation time measurements in coupled rooms," J. Acoust. Soc. Am. 3, 181-206(1931).

${ }^{9}$ L. Cremer and H. A. Muller, Principles and Applications of Room Acoustics, Vol. 1 (T. J. Schultz. Applied Science, New York, 1982), pp. 261276.

${ }^{10}$ H. Kuttruff, Room Acoustics, 4th ed. (Applied Science Publishers Ltd, London, 2000), Chap. 5, pp. 154-158.

${ }^{11}$ J. S. Anderson, M. Bratos-Anderson, and P. Doany, "The acoustics of a large space with a repetitive pattern of coupled rooms," J. Sound Vib. 208, 313-329 (1997)

${ }^{12}$ J. E. Summers, R. R. Torres, and Y. Shimizu, "Statistical-acoustics models of energy decay in systems of coupled rooms and their relation to geometrical acoustics," J. Acoust. Soc. Am. 116, 958-969 (2004).

${ }^{13}$ Y. Jing and N. Xiang, "Visualizations of sound energy across coupled rooms using a diffusion equation model," J. Acoust. Soc. Am. 124, EL360-EL365 (2008).

${ }^{14}$ C. D. Lyle, "An improved theory for transient sound behaviour in coupled diffused spaces," Acoust. Lett. 4, 248-252 (1981).

${ }^{15}$ C. M. Harris and H. Feshbach, "On the acoustics of coupled rooms," J. Acoust. Soc. Am. 22, 572-578 (1950).

${ }^{16} \mathrm{C}$. Thompson, "On the acoustics of a coupled space," J. Acoust. Soc. Am. 75, 707-714 (1984). 
${ }^{17}$ J. B. Allen and D. A. Berkley, "Image method for efficiently simulating small-room acoustics," J. Acoust. Soc. Am. 65, 943-950 (1979).

${ }^{18}$ A. Krokstad, S. Strom, and S. Sorsdal, "Calculating the acoustical room response by the use of a ray tracing technique," J. Sound Vib. 8, 118-125 (1968).

${ }^{19}$ G. M. Naylor, "Odeon - Another hybrid room acoustical model," Appl. Acoust. 38, 131-143 (1993).

${ }^{20} \mathrm{M}$. Vorländer, "Simulation of the transient and steady-state sound propagation in rooms using a new combined ray-tracing/image-source algorithm," J. Acoust. Soc. Am. 86, 172-178 (1989).

${ }^{21}$ A. Billon, V. Valeau, A. Sakout, and J. Picaut, "On the use of a diffusion model for acoustically coupled rooms," J. Acoust. Soc. Am. 120, 2043-2054 (2006).

${ }^{22}$ Y. Jing and N. Xiang, "A modified diffusion equation for room-acoustic predication (L)," J. Acoust. Soc. Am. 121, 3284-3287 (2007).

${ }^{23}$ V. Valeau, J. Picaut, and M. Hodgson, "On the use of a diffusion equation for room-acoustic prediction,” J. Acoust. Soc. Am. 119, 1504-1513 (2006).
${ }^{24}$ N. Xiang and P. M. Goggans, "Evaluation of decay times in coupled spaces: Bayesian parameter estimation,” J. Acoust. Soc. Am. 110, 14151424 (2001)

${ }^{25}$ M. Ermann and M. Johnson, "Exposure and materiality of the secondary room and its impact on the impulse response of coupled-volume concert halls," J. Sound Vib. 284, 915-931 (2005).

${ }^{26}$ M. R. Schroeder, "New method of measuring reverberation time," J. Acoust. Soc. Am. 37, 409-412 (1965).

${ }^{27}$ D. He, "An overview of microflown technologies," Acta Acust. Acust. 89, 167-172 (2003).

${ }^{28}$ F. J. Fahy, Sound Intensity, 2nd ed. (E \& FN Spon, London, 1995), Chap. 5, pp. 89-99.

${ }^{29}$ N. Xiang and P. M. Goggans, "Evaluation of decay times in coupled spaces: Bayesian decay model selection," J. Acoust. Soc. Am. 113, 26852697 (2003). 